\title{
Modulating Electron Density in the Bound Product, 4-Hydroxybenzoyl-CoA, by Mutations in 4-Chlorobenzoyl-CoA Dehalogenase Near the 4-Hydroxy Group ${ }^{\dagger}$
}

\author{
Jian Dong, ${ }^{\ddagger}$ Hong Xiang, ${ }^{\S}$ Lusong Luo, ${ }^{\S}$ Debra Dunaway-Mariano, ${ }^{\S}$ and Paul R. Carey*,\$ \\ Department of Biochemistry, Case Western Reserve University, 10900 Euclid Avenue, Cleveland, Ohio 44106, and Department \\ of Chemistry, University of New Mexico, Albuquerque, NM 87131
}

Received November 9, 1998; Revised Manuscript Received February 4, 1999

\begin{abstract}
The enzyme 4-chlorobenzoyl-CoA dehalogenase hydrolyzes 4-chlorobenzoyl-CoA (4-CBACoA) to 4-hydroxybenzoyl-CoA (4-HBA-CoA). Biochemical and crystallographic studies have identified a critical role for the dehalogenase residue Asp 145 in close proximity to the ligand's 4-hydroxy group in the structure of the product-enzyme complex. In the present study the effects of site selective mutations at Asp 145 on the product complex are explored by Raman spectroscopy. The spectral signatures of the WT-product complex, the large red shift in $\lambda_{\max }$, and the complete reorganization of the benzoyl ring modes in Raman data are absent for the D145E complex. The major spectral perturbations in the WT complex are brought about by strong electron "pull" at the benzoyl carbonyl and electron "push" by the side chain of Asp 145 near the 4-OH group. Acting in concert, these factors polarize the benzoyl's $\pi$-electrons. Since the Raman data show that very strong electron pull occurs at the benzoyl's carbonyl in the D145E complex, it is apparent that the needed electron push near the benzoyl's 4-OH group is missing. Thus, very precise positioning of Asp 145's side chain near the benzoyl's 4-position is needed to bring about the dramatic electron reorganization seen in the WT complex, and this criterion cannot be met by the glutamate side chain with its additional $\mathrm{CH}_{2}$ group. For two other Asp145 mutants D145A and D145S that lack catalytic activity, Raman difference spectroscopic data for product complexes demonstrate the presence of a population of ionized product (i.e., 4- ${ }^{-}$) in the active sites. The presence of the ionized phenolate form explains the observation that these complexes have highly red-shifted absorbance maxima with $\lambda_{\operatorname{maxs}}$ near $400 \mathrm{~nm}$. For the WT complex only the 4-OH form is seen, ionization being energetically expensive with the presence of the proximal negative charge on the Asp 145 side chain. Semiquantitative estimates of the $\mathrm{p} K_{\mathrm{a}}$ for the bound product in D145S and D145A indicate that this ionization lies in the $\mathrm{pH} 6.5-7.0$ range. This is approximately $2 \mathrm{pH}$ units below the $\mathrm{p} K_{\mathrm{a}}$ for the free product. The Raman spectrum of 4-dimethylaminobenzoyl-CoA undergoes major changes upon binding to dehalogenase. The bound form has two features near 1562 and $1529 \mathrm{~cm}^{-1}$ and therefore closely resembles the spectrum of product bound to wild-type enzyme, which underlines the quinonoid nature in these complexes. The use of a newly developed Raman system allowed us to obtain normal (nonresonance) Raman data for the dehalogenase complexes in the 100-300 $\mu \mathrm{M}$ range and heralds an important advance in the application of Raman spectroscopy to dilute solutions of macromolecules.
\end{abstract}

4-Chlorobenzoyl-coenzymeA dehalogenase catalyzes the hydrolytic dehalogenation of 4-chlorobenzoyl-coenzyme A (4-CBA-CoA) to 4-hydroxybenzoate-CoA (4-HBA-CoA). This enzyme has been discovered in a number of soildwelling bacterial strains where it functions along with 4-CBA-CoA ligase and 4-HBA-CoA thioesterase to convert para-chlorobenzoic acid to para-hydroxybenzoic acid (for a review see Dunaway-Mariano and Babbitt, ref 1). Studies of the Pseudomous sp. strain CBS3 4-CBA-CoA dehalogenase have shown that it utilizes a unique form of catalysis in which an active site carboxylate bonds to the $C(4)$ of the benzoyl ring of the bound substrate to form a Meisenheimer complex which then proceeds to product through chloride

\footnotetext{
This work was supported by NIH Grants GM-54072 (to P.R.C.) and GM-28688 (to D.D.M.).

* To whom correspondence should be addressed. E-mail: carey@ biochemistry.cwru.edu. Fax: (216) 3683419.

$\stackrel{+}{*}$ Case Western Reserve University.

$\S$ University of New Mexico.
}

ion expulsion, the formation of an arylated intermediate, and hydrolysis as seen in Scheme $1(2,3)$.

The nature of the active site forces which increase the electrophilic character of the $\mathrm{C}(4)$-position is of interest since the formation of a Meisenheimer-like complex in the reaction is not chemically facile $(4,5)$. An indication that strong electrostatic forces are present in the active site came from absorption, Raman and NMR spectroscopic studies of enzyme complexes involving the product, and separately, a substrate analogue (6). The spectroscopic data showed that electron-polarizing forces can be brought to bear in the active site, which, in the case of the bound product, cause a major rearrangement of the electrons in the benzoyl moiety. Interest in these findings has recently been heightened by the publication of the structure of the dehalogenase-4-HBACoA complex derived by X-ray crystallography (7), and the $\mathrm{X}$-ray results have been used to analyze shifts in absorption maxima for ligand-enzyme complexes involving several 
Scheme 1: Reaction Pathway of 4-Chlorobenzoyl CoA Dehaogenase-Catalyzed Hydrolysis

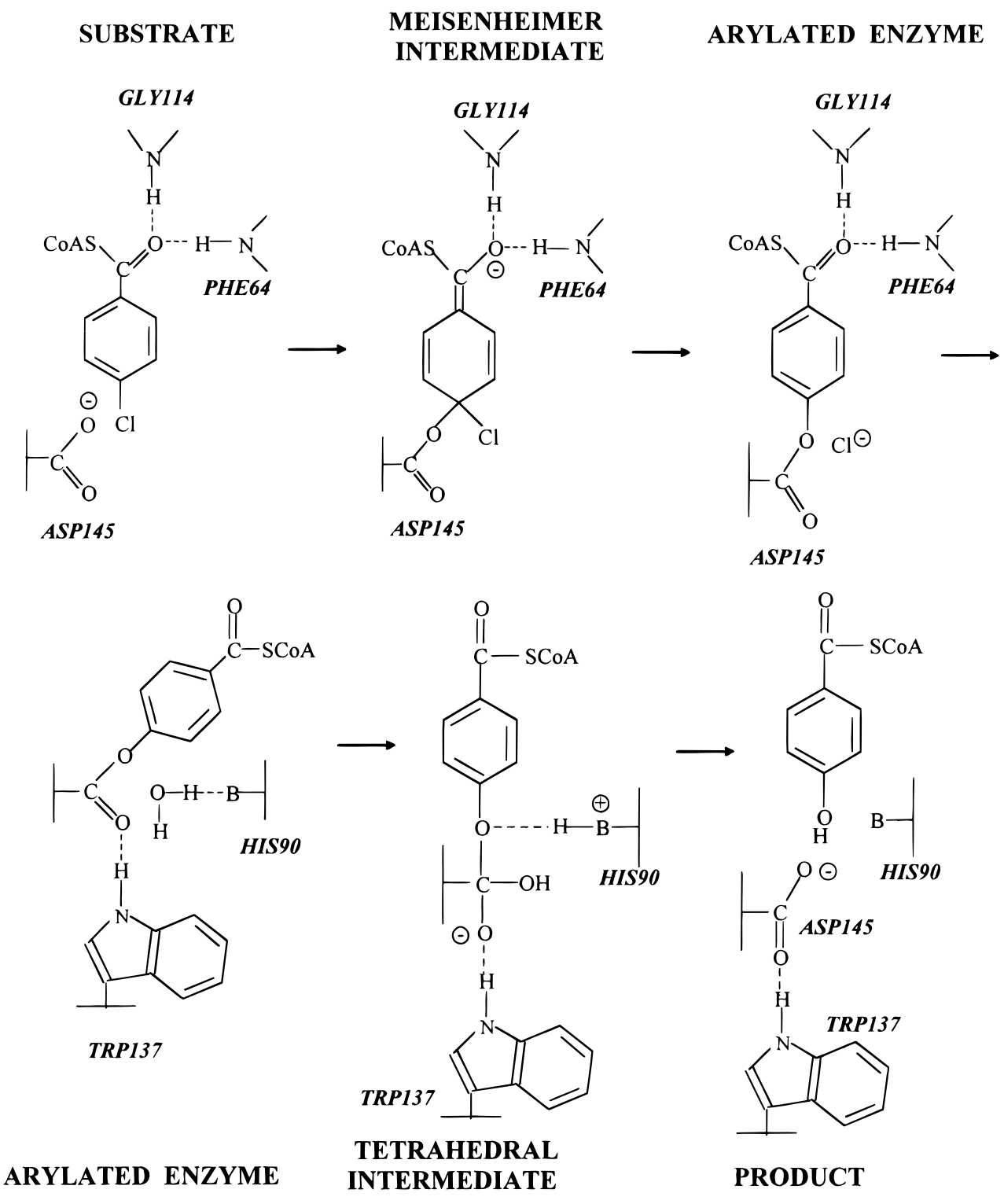

site-selected mutants (8). Recently, we published an analysis of the polarizing forces in the active site, combining Raman spectroscopic data on the bound product, including results on ${ }^{13} \mathrm{C}$ and ${ }^{18} \mathrm{O}$ substituted isotopomers of the product, with structural conclusions culled from the X-ray crystallographic studies (9). The spectral characteristics of strong polarization seen for the bound product are a large shift in $\lambda_{\max }$, from 292 to $370 \mathrm{~nm}$, and a dramatic rearrangement of the Raman normal modes of the benzoyl ring $(6,9)$. The conclusions from the combined Raman and X-ray data were as follows:

(i) Strong electron pull is exerted on the benzoyl carbonyl group by hydrogen bonds from the peptide NHs of Gly 114 and Phe 64. This carbonyl polarization effect is accentuated by the electrostatic field due to the $\alpha$-helix which has its N-terminus at Gly 114. The total polarizing effects of the hydrogen bonds and the $\alpha$-helix are equivalent to an effective hydrogen bonding strength of $57 \mathrm{~kJ} \mathrm{~mol}^{-1}$. It is noteworthy that similar polarizing effects may be operating in cysteine proteases to stabilize charge build-up in the transition state (10).

(ii) For product binding in the active site of the WT enzyme, a major rearrangement of the benzoyl $\pi$-electrons occurs. The entire $\pi$-system becomes highly polarized, with an incremental positive charge near the 4-position and an incremental negative charge on the benzoyl carbonyl oxygen.

(iii) Polarization effects throughout the benzoyl's $\pi$-electrons are assisted by the active site environment about the benzoyl group. The benzoyl group is encased in a sheath of aromatic amino acid side chains. Thus, the benzoyl is akin to a $\pi$-electron wire surrounded by a low dielectric insulator.

Undoubtedly, the above polarizing effects are "designed into" the active site in order to facilitate the difficult chemistry of the aromatic substitution of the 4- $\mathrm{Cl}$ group by the 4-OH group.

As already noted, binding of the product to dehalogenase's active site results in large perturbations to absorption and Raman spectral properties. The X-ray structure of the complex revealed that the side chain of Asp 145 is close to the 4-OH group (2.4 $\AA$ ) (7). It is likely that the formally negative $\mathrm{Asp}-\mathrm{COO}^{-}$and neutral product $4-\mathrm{OH}$ groups share the protium atom in an as yet ill-defined manner and this may impact on the observed spectral properties. In this paper we explore the role of Asp 145 by mutating the residue to glutamate, serine, and alanine. The results emphasize the 
critical nature of the interactions involving Asp 145 in the active site. Moreover, the discovery that significant amounts of ionized product can exist bound to the D145A and D145S mutants sets the scene for the accompanying publication (11) that discusses reactions in the active site involving the ionized product.

In two earlier publications dealing with dehalogenase (6, 9), we were able to obtain Raman difference spectroscopic data for ligands bound to the enzyme using a $0.5 \mathrm{~m}$ spectrograph and a charge-coupled device detector (12). This instrumentation was assembled in 1992 and represented significant improvements in sensitivity and fluorescence rejection compared to extant technology. However, even with these improvements, it was necessary to work at dehalogenase concentrations close to $1 \mathrm{mM}$ under normal (nonresonance) Raman excitation. It proved to be very difficult to obtain stable dehalogenase solutions at these concentrations. However, we have recently modified an f 1.4 spectrograph $(0.085 \mathrm{~m})$, with a holographic transmission grating, to yield a system of greater sensitivity (13). The new system enables us to work in the protein concentration range of 100-300 $\mu \mathrm{M}$ and has thus opened the way for a wide range of Raman studies of hitherto inaccessible biological systems. This and the following paper (11) represent our first demonstration of the power of the new Raman spectrometer.

\section{MATERIALS AND METHODS}

Sample Preparation. Pseudomonas sp. strain CBS3 4-CBACoA dehalogenase was isolated from the Escherichia coli expression system (14) as described in Liang et al. (15). The 4-CBA-CoA dehalogenase mutants D145E, D145A, and D145S were prepared as described in Xiang (16). 4-HBACoA was prepared using the procedure of Mieyel et al. (17) as modified in Liang et al. (15). The wild-type and mutant enzymes were concentrated to $\sim 100 \mu \mathrm{M}$ before use. For all of the dehalogenase complexes studied in this paper, the $K_{\mathrm{d}}$ values are less than $1 \mu \mathrm{M}$ (see page 70 of ref 16 ); thus all spectral data are for the bound form of the ligands.

Raman Spectroscopy. Raman spectra were obtained using $647.1 \mathrm{~nm}$ laser excitation from an Innova 400 krypton laser system (Coherent, Inc.) and a back-illuminated CCD detector (Model 1024EHRB/1, Princeton Instruments, Inc.) operating at $183 \mathrm{~K}$. A modified Holospec f/1.4 axial transmission spectrometer (Kaiser Optical Systems, Inc.) equipped with a holographic Supernotch-Plus filter and a transmissive holographic VPT grating was employed as a single monochromator (13). Enzyme samples, $60 \mu \mathrm{L}$ and buffered with $50 \mathrm{mM}$ Tris- $\mathrm{HCl}$ at $\mathrm{pH} 7.5$, were contained in glass cuvettes. During experiments, the concentration of the "free" enzyme ought to be made identical to the enzyme concentration in the complex to circumvent the introduction of a subtraction factor. After the Raman spectrum of the enzyme was obtained, 4-HBA-CoA was added in a quantity equal to the active site concentration of enzyme, and the data were collected immediately after the complex had been made, under a laser power of $\sim 850 \mathrm{~mW}$ with a CCD exposure time of $5 \mathrm{~min}$. The Raman spectrum of the buffer was subtracted from that of the ligand in buffer (giving the spectrum of free ligand), while the spectrum of the enzyme in buffer was subtracted from that of the enzyme-ligand complex in buffer (giving the spectrum of the enzyme-bound ligand). The subtraction of data sets was performed using GRAMS/32 (Galactic Industries, Inc., New Hampshire) software.

Several factors contribute to the stability of our Raman instrumentation and assist in undertaking accurate subtraction experiments. Neither the sample arrangement nor any optical component (lenses or grating) inside the spectrograph moves during data acquisition $(12,13)$. This is important to ensure that the images of the two parent spectral signals do not undergo a displacement with respect to each other. The subtraction process is facilitated further by the fact that the ligands are fairly strong Raman scatterers. At a laser power of $0.850 \mathrm{~W}$ and an accumulation time of $5 \mathrm{~min}$, a D145A mutant enzyme $(270 \mu \mathrm{M})$ has a noise amplitude of 21000 counts, while the free product can generate a signal of $\sim 363000$ counts for a concentration of $270 \mu \mathrm{M}, 18$ times larger than the noise (an identifiable peak usually requires a $\mathrm{S} / \mathrm{N}$ ratio of 3). Crucially our confidence in the quality of the Raman difference data is based on the reproducibility of the data from the single-chamber spectrographs. For example, the first Raman difference data for product bound to WT dehalogenase were generated in Ottawa, Canada, using a Spex $0.5 \mathrm{~m}$ spectrograph (12) in 1993 (6). They are identical, in every respect, to those obtained in Cleveland in 1998 using a modified spectrograph $(0.085 \mathrm{~m})$ based on a Kaiser instrument "Holospec" (13). The recent high through-put holographic technology and NIR-optimized CCD detection both improve the sensitivity remarkably; thus the data collection time has been reduced to only a few minutes. This compares to conventional methods carried out using a double (or triple) monochromator and photodiode array detectors that usually require data summation for an hour or so.

Vibrational Analysis. Harmonic frequency calculations of $S$-ethyl 4-hydroxybenzoate thioester, $S$-ethyl 4-oxybenzoate thioester anion, and $S$-ethyl 4-dimethylaminobenzoate thioester were carried out with the Gaussian 94 package (18) on a T90 vector processor (Ohio Supercomputer Center, Columbus) or a Silicon Graphics workstation in this laboratory. The geometry of each molecule was completely optimized at the appropriate level of theory by analytic gradient techniques. A density functional theory (DFT) variant B3LYP with a basis set of 6-31+G(d) was first employed to give normal modes. Then, a Hartree-Fock (HF) method with the same basis set was performed, producing Raman activities and nearly the same normal modes as those from DFT level. The calculated frequencies at the DFT/B3LYP level were converted by a single scaling factor (0.9692) through a least-squares approach, according to a recent comprehensive evaluation of Scott and Radom (19). Normal modes related to ring vibrations (in Wilson's notation (20)) are described according to Colthup et al. (21) and Dollish et al. (22).

\section{RESULTS AND DISCUSSION}

Band Assignments for 4-HBA-CoA. The Raman spectra of 4-HBA-CoA in its phenol form, at $\mathrm{pH} 7.5$, and its phenolate form, at $\mathrm{pH}$ 10.5, are shown in Figure 1. The results of the quantum mechanical calculations (examined by both density functional theory and an ab initio MO method) are given in Tables 1 and 2 (Table 2 appears in Supporting Information) for the protonated and ionized forms of 4-HBA-ethylthioester, respectively (to make the calcula- 


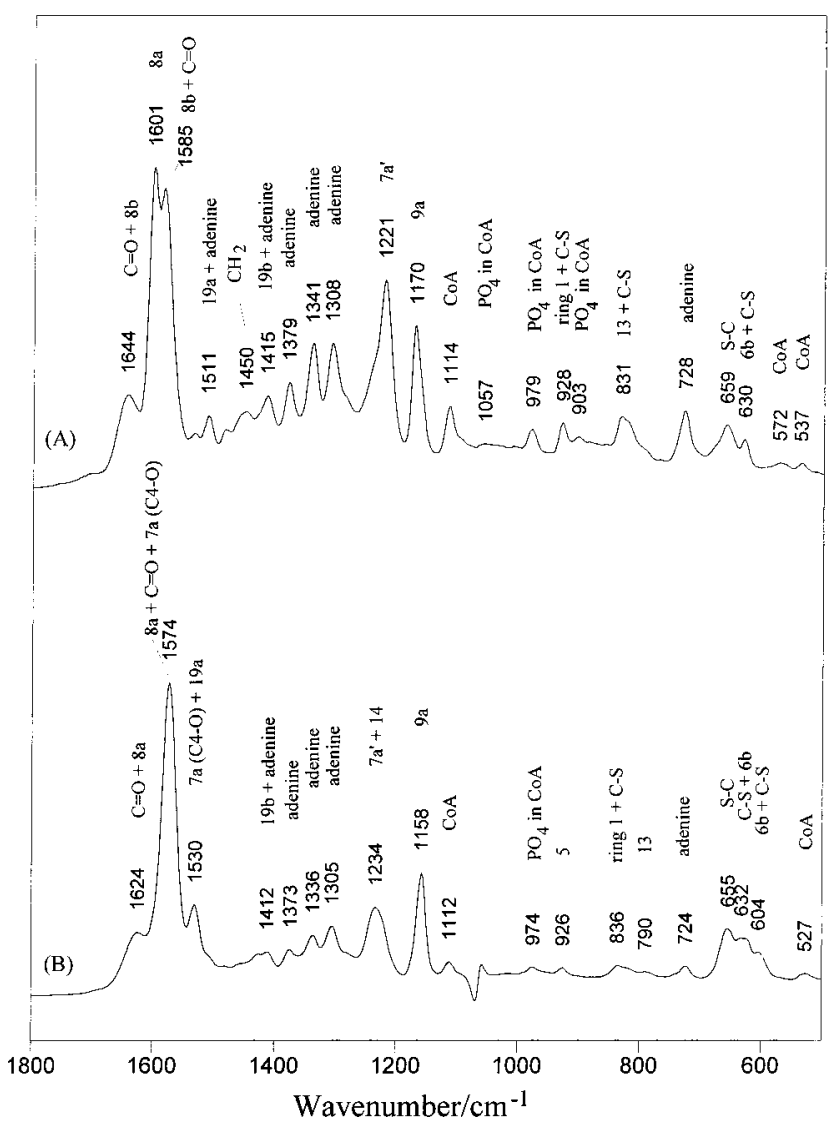

FIGURE 1: Raman spectra of free product 4-hydroxybenzoyl CoA (4-HBA-CoA): (A) $8 \mathrm{mM}$ concentration, in $50 \mathrm{mM}$ Tris- $\mathrm{HCl}$ buffer solution, $\mathrm{pH} 7.5$; (B) $10.1 \mathrm{mM}$ concentration, in $100 \mathrm{mM} \mathrm{Na}_{2} \mathrm{CO}_{3}-$ $\mathrm{NaHCO}_{3}$ buffer solution, $\mathrm{pH}$ 10.5. (The negative band near 1050 $\mathrm{cm}^{-1}$ is a $\mathrm{CO}_{3}^{2}$-band in the buffer).

tions tractable, the CoA moiety is replaced by an ethyl group). The calculations, taken with our previous Raman work on $S$-ethyl 4-hydroxybenzoate thioester (6) and isotopically labeled 4-HBA-CoA (9), published work on benzene ring (23) and adenine modes $(24,25)$, and unpublished work in this laboratory on CoA and adenosine compounds enable us to assign most of the Raman features in Figure 1 with confidence. The most intense features in the spectra, as well as several minor bands, are due to benzene ring modes, and these are identified in Figure 1 by the numerical alphabet notation of Wilson (20). For most of the dehalogenaseligand complexes discussed in this series of papers, the benzene ring modes appear close to the positions seen in Figure 1 and only undergo large changes when there is a major redistribution of electron density within the $\pi$-electron system. Features from the thioester moiety are also observed; most notably the band near $1630 \mathrm{~cm}^{-1}$ has $\mathrm{C}=\mathrm{O}$ stretching character, while the band near $660 \mathrm{~cm}^{-1}$ has a major contribution from the $\mathrm{S}-\mathrm{C}$ stretch from the $\mathrm{C}(=\mathrm{O}) \mathrm{S}-\mathrm{C}$ bond. Bands from the CoA cofactor emanate from adenine ring modes or the phosphate groups, and these are displayed in Figure 1. The former give rise to a series of sharp, resolved bands that are seen in many of the Raman difference spectra of dehalogenase-ligand complexes.

When the phenolic form is changed into the phenolate form, the negative charge is mainly localized on the oxygen substituent and adjacent ring C(4) atom, rather than on other ring carbon atoms, as revealed by DFT and HF calculations (unpublished work, this laboratory). Accordingly, the fre-

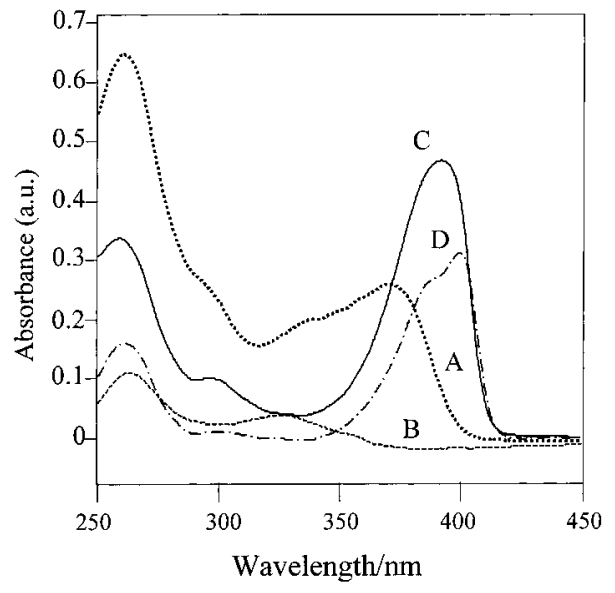

FIGURE 2: UV-Vis absorbance spectra of 4-HBA-CoA bound in (A) WT $(15 \mu \mathrm{M})$ (dotted line); (B) D145E $(8 \mu \mathrm{M})$ (dashed line); (C) D145S $(16 \mu \mathrm{M})$ (solid line); and (D) D145A (11 $\mu \mathrm{M})$ dehalogenase (dash-dotted line). The enzyme to 4-HBA-CoA concentration ratio is $1: 1$, except for $(\mathrm{B})$ where it is $2: 1$. Enzyme samples (in $50 \mathrm{mM}$ Tris- $\mathrm{HCl}$ buffer, $\mathrm{pH}$ 7.5) with identical concentrations were taken as reference.

quency of the $\mathrm{C}(4)-\mathrm{O}$ stretching vibration (ring mode $7 \mathrm{a}$ ) is significantly raised to about $1530 \mathrm{~cm}^{-1}$ and couples with the ring 19a mode, but remains lower than the "normal" $\mathrm{C}=$ $\mathrm{O}$ stretching frequency. Other accompanying changes in benzoyl ring modes (e.g., 8a, 7a', 9a, and 1) are straightforward and are illustrated in Figure 1. In toto, the above Raman features constitute a rich source of information on changes occurring in 4-X-benzoyl-CoA (where X can be one of a number of substituents in the 4-position) when this ligand binds to dehalogenase or to one of the protein-engineered forms of this enzyme.

Comparison of 4-HBA-CoA Bound to WT and D145E Dehalogenase. The structure of 4-HBA-CoA bound to WT dehalogenase has been determined to $1.8 \AA$ resolution by X-ray crystallography (7). The CoA ligand is bent back upon itself to the degree that hydrogen bonding contacts involving the backbone $\mathrm{C}=\mathrm{O}$ and $\mathrm{NH}$ of Phe 64 are made to the adenine residue at one end of the CoA and to the benzoyl carbonyl group at the other end. A major theme in the present series of studies involves mutations at aspartate 145. Seen in Scheme 1, this residue plays a key role in the reaction. Replacement by any other amino acid results in an inactive enzyme; the exception is D145E which, for the reaction with substrate, has a $k_{\text {cat }}$ of $0.001 \mathrm{~s}^{-1}$, which is 600 times slower than for WT.

Free 4-HBA-CoA at $\mathrm{pH} 7.5$ has a $\lambda_{\max }$ at $292 \mathrm{~nm}$ with a shoulder near $335 \mathrm{~nm}(6)$; when 4-HBA-CoA binds to WT, $\lambda_{\max }$ shifts to $373 \mathrm{~nm}$ with a shoulder near $330 \mathrm{~nm}$ (Figure 2) (6). In contrast, when 4-HBA-CoA binds to D145E, the $\lambda_{\max }$ occurs at $330 \mathrm{~nm}$ (Figure 2). The large differences between the absorption spectroscopic properties of the product bound to D145E and WT enzyme, as well as the differences in reactivity, suggest that the positioning of the aspartate 145 side chain is critical. This conclusion is fully substantiated by the Raman data shown in Figure 3; the latter compares the Raman spectrum of the free product $(4-\mathrm{OH}$ form) with those of the product bound to WT and D145E dehalogenase. The data for the 4-HBA-CoA binding to WT have been discussed in earlier publications, where the analyses were assisted by ${ }^{13} \mathrm{C}$ and ${ }^{18} \mathrm{O}$ substitutions in the 


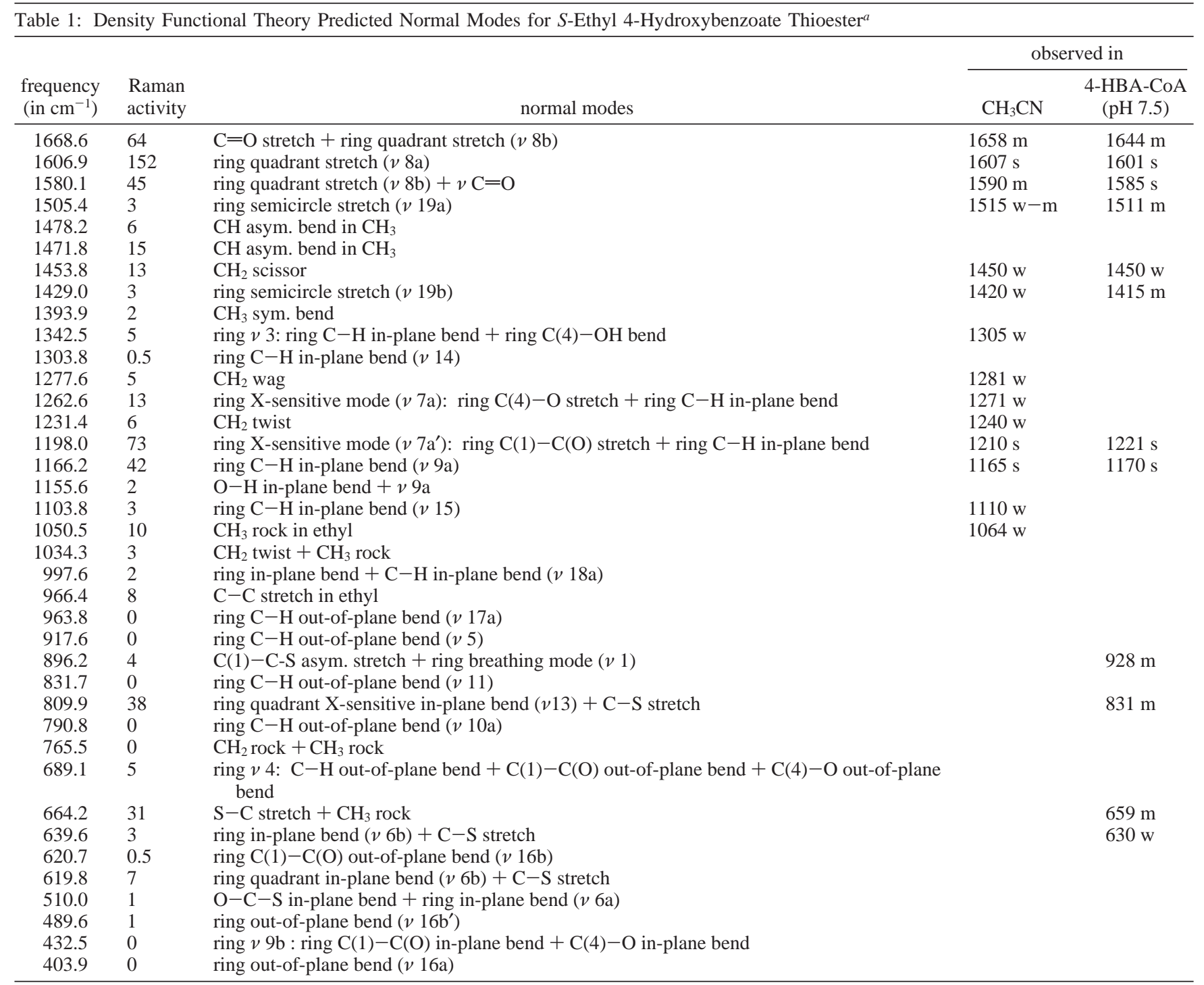

${ }^{a}$ Calculated Raman activity values less than 0.5 are denoted 0 . The experimental data of $S$-ethyl 4-hydroxybenzoate thioester in acetonitrile are available in ref 6 as Supporting Information. Also see P.-H. Liang, Ph.D. thesis, p 79, University of Maryland, 1994. Abbreviations for observed intensity: s, strong; m, medium; w, weak; br, broad.

benzoyl $\mathrm{C}=\mathrm{O}$ and $4-\mathrm{OH}$ groups $(6,9)$. A key conclusion from these studies was that, for the WT complex, major $\pi$-electron reorganization occurs for the benzoyl moiety with the ring becoming more quinonoid-like than benzenoid-like. Thus, valence structures such as II make important contributions to the structure in the active site (see Scheme 2).

As a result of the $\pi$-electron reorganization, the normal mode makeup of the benzene ring modes changes and the Wilson-type assignments no longer obtain. Thus, the isotopic substitutions demonstrated that the strong benzoyl ring features at 1561 and $1526 \mathrm{~cm}^{-1}$ in Figure 3B contain important contributions from motions of the benzoyl $\mathrm{C}=\mathrm{O}$ group. The agents bringing about this electron reorganization are strong electron "pull" at the carbonyl from hydrogen bonds and the $\alpha$-helix dipole (which runs Gly114 to Ala121), electron "push" from the negatively charged side chain of Asp 145 at the 4-hydroxy group, and the low dielectric environment about the bound benzoyl ring caused by the sheath of aromatic side chains (9).

There is marked contrast between the Raman data for the product bound to WT and D145E. As is seen in Figure 3C the key benzene ring modes near 1600, 1582, 1220, and 1169 $\mathrm{cm}^{-1}$ for the free product remain "intact" for the 4-HBACoA-D145E complex. Thus, major $\pi$-electron reorganization has not occurred within the benzoyl moiety. However, strong polarization of the carbonyl is present; the $\mathrm{C}=\mathrm{O}$ feature cannot be detected in Figure 3 because it has moved to lower wavenumbers and is either obscured "under" the ring modes $8 \mathrm{a}$ and $8 \mathrm{~b}$ or has vibrationally coupled with them. Since strong polarization is present at the carbonyl and we presume that the benzoyl ring is still encapsulated in its aromatic side chain sheath, the difference between the data for the WT and D145E complexes must reside in the group close to the 4-position of the benzene ring. The glutamate side chain, with its extra $\mathrm{CH}_{2}$ group, compared to aspartate, is unable to interact significantly near the 4-OH-position to promote the kind of valence structure shown in Scheme 2. Since the structure of the D145E-product complex has not been solved by X-ray crystallographic analysis, we do not know the position of the glutamate side chain with respect to the 4-position of the ring. However, the present findings emphasize that the steric requirements for the Asp 145- 
Scheme 2: Electron Redistribution of Product (4-HBA-CoA) in Wild-Type Dehalogenase (Structure I and II) ${ }^{a}$

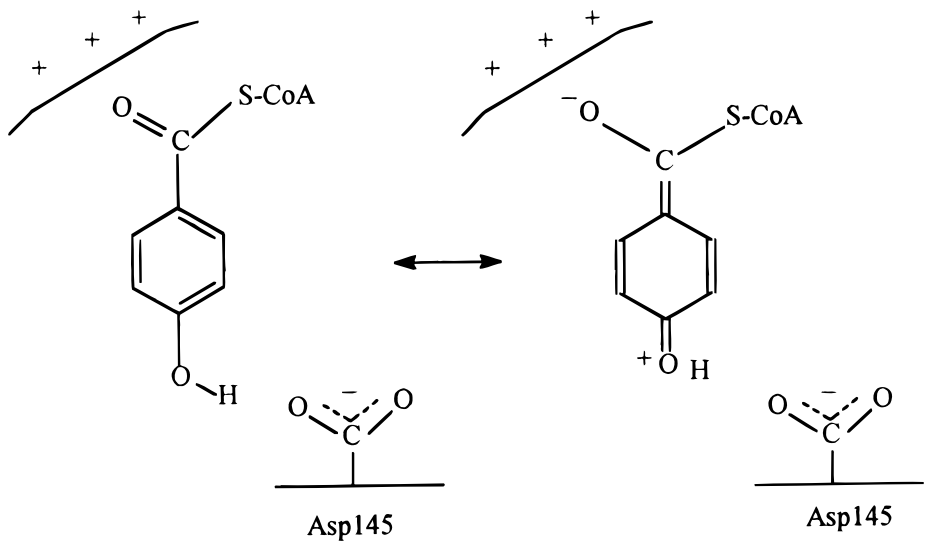

(I)
(II)

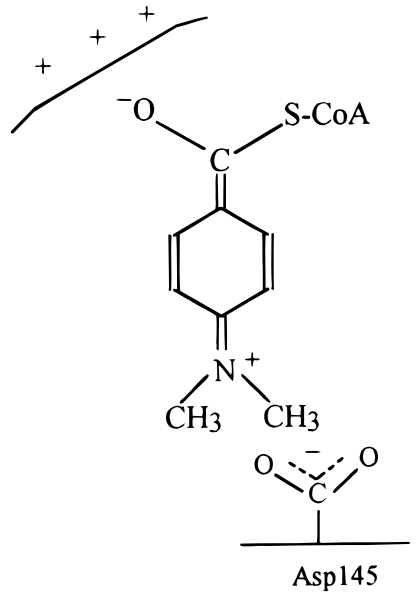

(III)

${ }^{a}$ The polarized form (II) represents the dominant contribution. The analogue 4-DMABA-CoA in the wild-type enzyme exhibits a similarly polarized form like (III) (see text). The positive signs represent $\mathrm{H}$-bond and $\alpha$-helix dipole forces adjacent to the $\mathrm{C}=\mathrm{O}$.

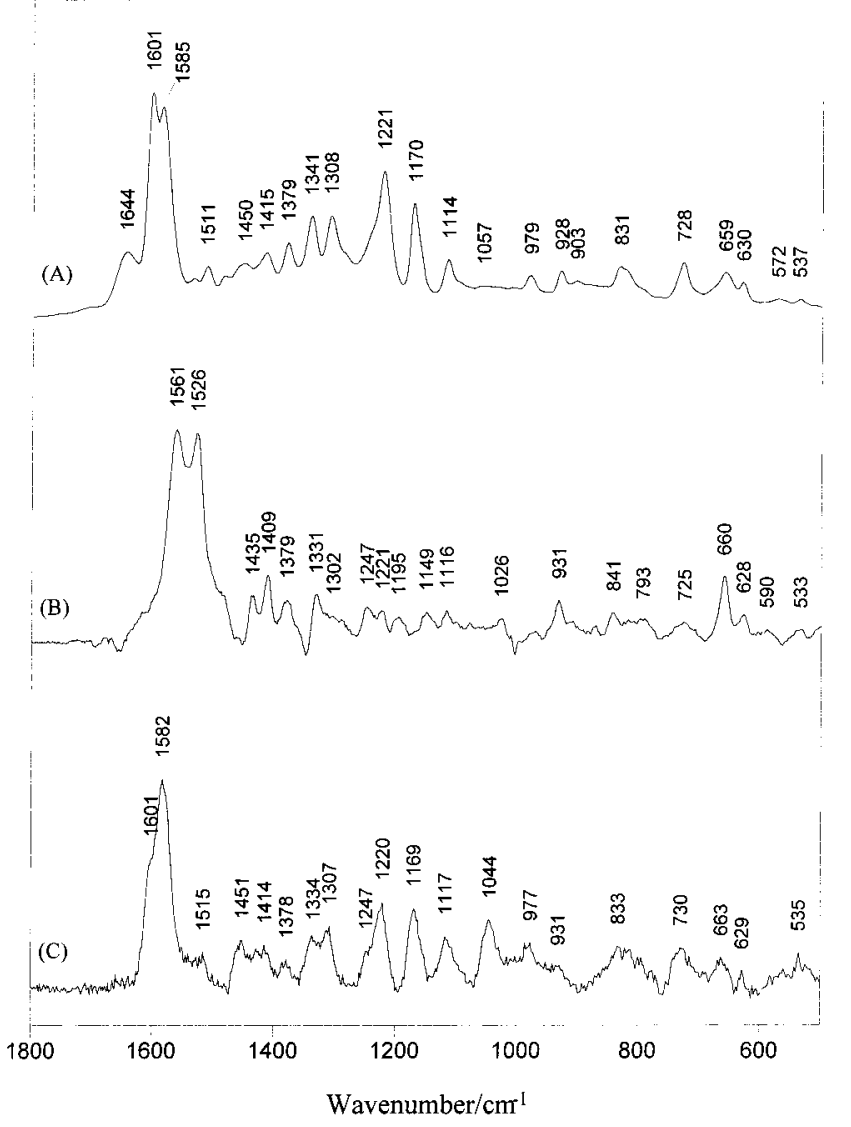

FIGURE 3: Raman spectra of 4-HBA-CoA: (A) free state, $8 \mathrm{mM}$, in $50 \mathrm{mM}$ Tris- $\mathrm{HCl}$ buffer, $\mathrm{pH} 7.5$; (B) bound to WT $(444 \mu \mathrm{M}$, in $50 \mathrm{mM}$ Tris-HCl buffer, $\mathrm{pH} 7.5$ ); (C) bound to the D145E mutant (120 $\mu \mathrm{M}$, in $50 \mathrm{mM}$ Tris- $\mathrm{HCl}$ buffer, $\mathrm{pH} 7.5)$.

benzoyl interaction near the 4-position are likely constrained within narrow limits in order to bring about electron polarization within the $\pi$-electron chain.

In the Raman spectrum of the D145E complex several features of minor intensity can be assigned by reference to the data given in Figure 1 and Table 1. The adenine "triplet" between 1300 and $1380 \mathrm{~cm}^{-1}$ shows a characteristic change upon binding that is repeated for many other complexes. One

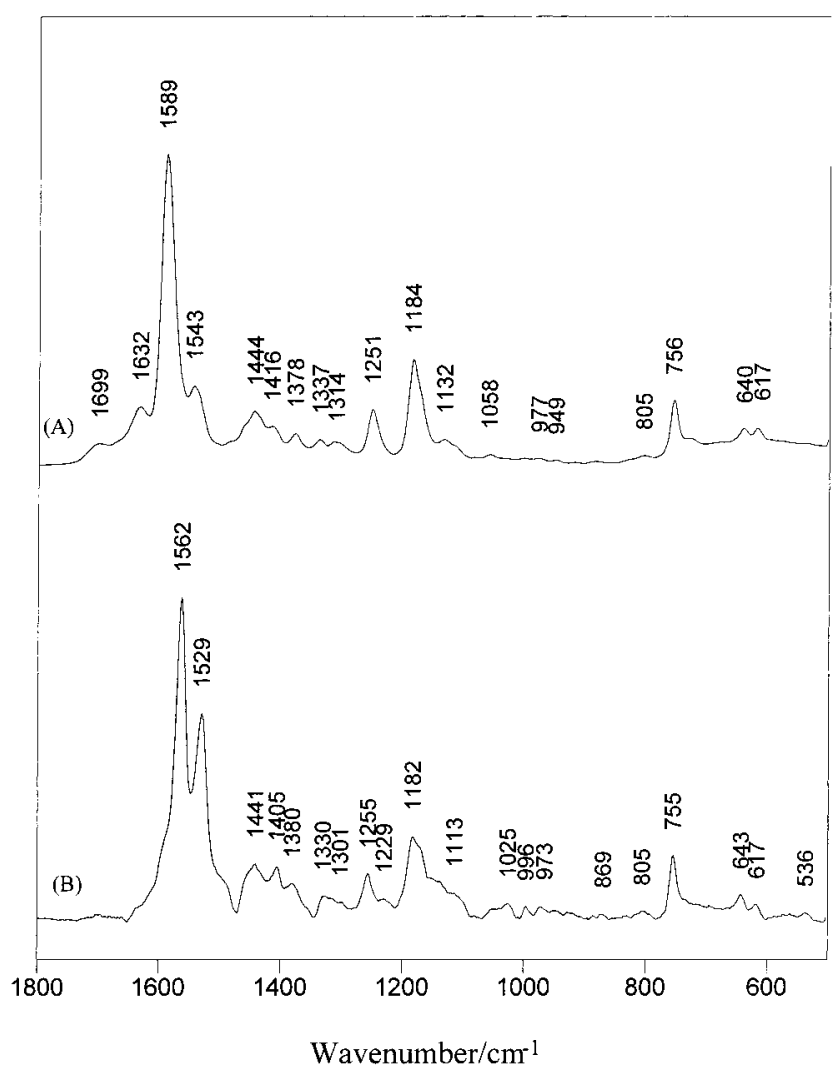

FIGURE 4: Raman spectra of (A) free 4-DMABA-CoA $(9.9 \mathrm{mM})$ in Tris buffer; (B) 4-DMABA-CoA ligand bound to WT $(180 \mu \mathrm{M}$ in $50 \mathrm{mM}$ Tris- $\mathrm{HCl}, \mathrm{pH} 7.5$ ).

feature that has not been assigned is the band occurring near $1045 \mathrm{~cm}^{-1}$. Bands in this region may be associated with the CoA's phosphate groups, but this feature is not observed for other complexes. It is noteworthy that there is little evidence for "negative" peaks in the Raman difference spectrum which means that the difference spectra are dominated by contributions from the bound ligand and that few changes in protein conformation, upon ligand binding, can be detected.

The data for the D145E complex establish the fact that the $330 \mathrm{~nm}$ absorption band is due to the bound ligand with 
strong polarizing forces acting on the benzoyl $\mathrm{C}=\mathrm{O}$ but lacking significant electron "push" at the para-position. Recalling that the absorption spectrum for the WT complex consists of a $\lambda_{\max }$ at $373 \mathrm{~nm}$ with a shoulder near $330 \mathrm{~nm}$ (Figure 2), this raises the interesting possibility that there are two conformational populations in the WT complex: a major population with the aspartate 145 side chain near the 4-position, giving rise to the absorption near $370 \mathrm{~nm}$, and a minor population that has the aspartate side chain rotated away from the 4-position that gives rise to the $330 \mathrm{~nm}$ band. The Raman spectra of a mixture of these complexes would be dominated by contributions from the $370 \mathrm{~nm}$ species since this is the most red-shifted and polarized form. The fact that a second population was not reported in the crystallographic studies could reflect the low population of the less-polarized form or the fact that only a single population exists in the crystalline state in contrast to two populations for the complex in solution.

4-Dimethylaminobenzoyl-CoA-Dehalogenase as a Model for Product Binding. Figure 4 compares the Raman spectra of 4-dimethylaminobenzoyl-CoA free in aqueous solution and bound to dehalogenase. The spectrum of the free compound is dominated by the usual phenyl ring modes, $8 \mathrm{a}$ and $8 b$, near $1589 \mathrm{~cm}^{-1}$ and $7 \mathrm{a}^{\prime}$ and $9 \mathrm{a}$ near 1251 and 1184 $\mathrm{cm}^{-1}$, respectively (detailed assignments according to density functional theory are listed in Table 3 in Supporting Information). However, for the bound form, the $8 \mathrm{a}$ and $8 \mathrm{~b}$ features are replaced by two intense bands near 1562 and $1529 \mathrm{~cm}^{-1}$, thus bearing a striking resemblance to the Raman spectrum of product binding to WT seen in Figure 3B. We ascribe this to the fact that there are close similarities in the $\pi$-electron distribution in the two forms, that is, form II and III depicted in Scheme 2.

The features near 1562 and $1529 \mathrm{~cm}^{-1}$ are no longer "standard" benzenoid modes. For the WT enzyme-product complex, both showed sizable, $15-20 \mathrm{~cm}^{-1}$ downshifts when bound product was labeled with ${ }^{13} \mathrm{C}$ or ${ }^{18} \mathrm{O}$ in the benzoyl carbonyl group (9). Thus, it is likely that the normal mode structure of the two intense features is highly delocalized, encompassing the $\mathrm{C}=\mathrm{O}$ bonds as well as the ring. However, the modes do not seem to be sensitive to the nature of the group in the 4-position. In the structures of II and III in Scheme 2, the aromatic moiety is neutral overall. When the structure loses a protium to become negative, as for product binding to the D145S or D145A variants, discussed in the next section, a different distribution of electrons occurs. The $1560,1525 \mathrm{~cm}^{-1}$ signature is replaced by features near 1590 , $1540,1490,1400,1260$, and $1150 \mathrm{~cm}^{-1}$.

Ionization of the Product's $4 \mathrm{OH}$ in the Active Sites of D145S and D145A. The reported absorption spectral data for product binding to D145S and D145A dehalogenase are atypical in that the $\lambda_{\max }$ values are considerably red-shifted, occurring at 399 and $398 \mathrm{~nm}$, respectively $(8,16)$, and the absorption spectra for these complexes are shown in Figure 2. The structure of the D145A complex has been solved by crystallography (private communication, $\mathrm{M}$. Benning and $\mathrm{H}$. Holden) and is very similar to that of the WT complex providing no clue to the origins of the red-shifted absorption maximum. However, the cause of the red shift is immediately apparent from the Raman data; for the D145S and D145A forms of dehalogenase, the 4-OH group can ionize giving rise to polarization over and above that seen for the un-ionized form in the WT active site.

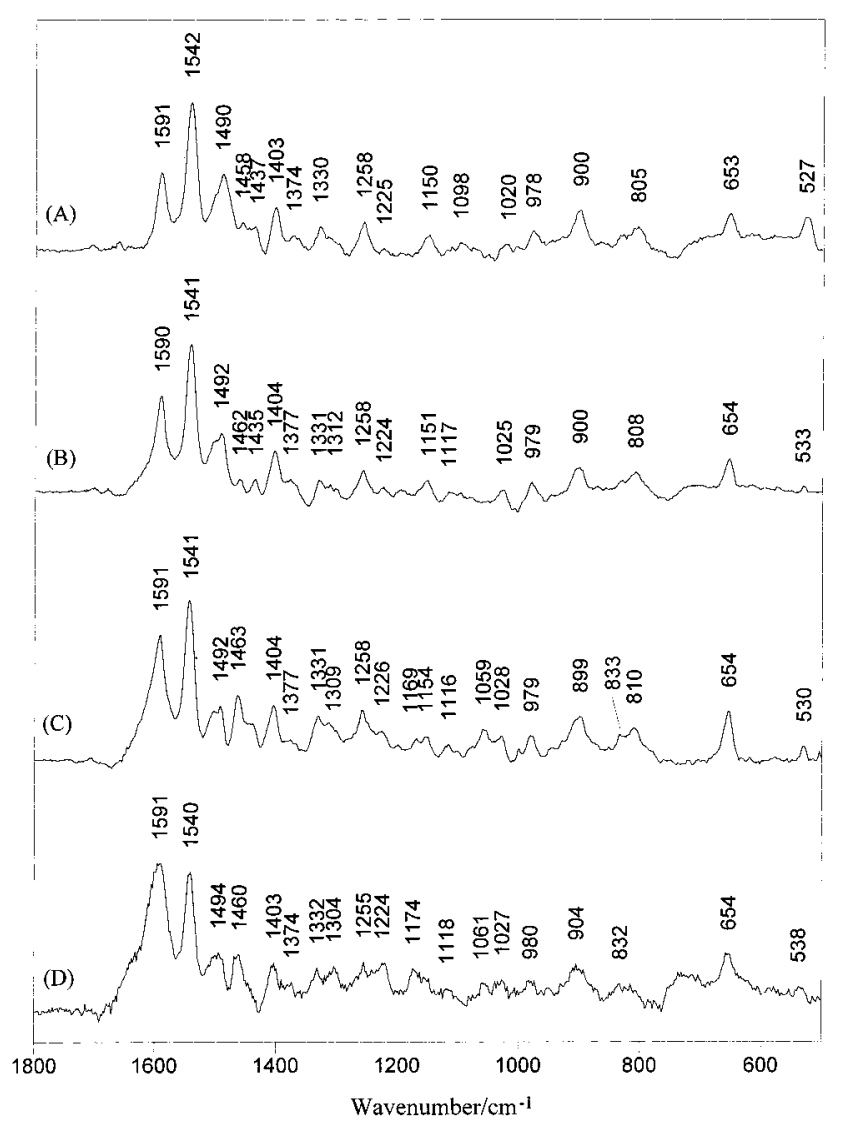

FIGURE 5: Raman spectra of 4-HBA-CoA bound to the D145S mutant at various pHs: (A) 8.0; (B) 7.5; (C) 7.0; and (D) 6.5. All of the spectra were taken in $50 \mathrm{mM}$ Tris- $\mathrm{HCl}$ buffer.

The Raman difference spectra for 4-HBA-CoA bound to $\mathrm{D} 145 \mathrm{~S}$ at $\mathrm{pH}$ values of 8.0, 7.5, 7.0, and 6.5 are compared in Figure 5. At the higher $\mathrm{pHs}$ the spectra are dominated by a series of bands near 1591, 1542, 1490, 1403, 1258, and $1150 \mathrm{~cm}^{-1}$ which have little or no correspondence with either phenolic or phenolate form of the free product, Figure 1, or with the product bound to WT, Figure 3B. However, at $\mathrm{pH}$ 6.5 the bands seen at higher $\mathrm{pH}$ are diminished and are replaced by ring modes seen for the nonpolarized phenolic ligand (4-OH form) in Figure 1A or Figure 3C, namely, the broad feature containing the $8 \mathrm{a}$ and $8 \mathrm{~b}$ modes near $1590 \mathrm{~cm}^{-1}$ and the $7 \mathrm{a}^{\prime}$ and 9a modes near 1224 and $1174 \mathrm{~cm}^{-1}$, respectively. At pH 6.5 a substantial amount of the $4-\mathrm{OH}$ form (nonpolarized) of the product is present in the active site and the phenolic species can still be detected at $\mathrm{pH} 7.0$ via the appearance of shoulders near 1226 and $1169 \mathrm{~cm}^{-1}$. However, at $\mathrm{pH} 7.5$ there is no spectral evidence for the 4-OH form and the observed spectrum is due to the deprotonated ligand. The Raman spectra of the free and bound phenolate species are distinct since in the bound form the strong electron pull at the benzoyl carbonyl gives rise to large contributions from a quinonoid-like valence bond structure (Scheme 3). The quinonoid structure resembles that proposed for the WT-product complex, but the electron distribution within them will be different due to the latter being neutral and that seen in Scheme 3 being negatively charged. The high degree of electron polarization seen in Scheme 3 accounts for the red shift seen in the absorption maximum; the $\lambda_{\max }$ for the D145S complex is at $399 \mathrm{~nm}$ compared to $373 \mathrm{~nm}$ for the WT complex and $330 \mathrm{~nm}$ for the free phenolate form. 


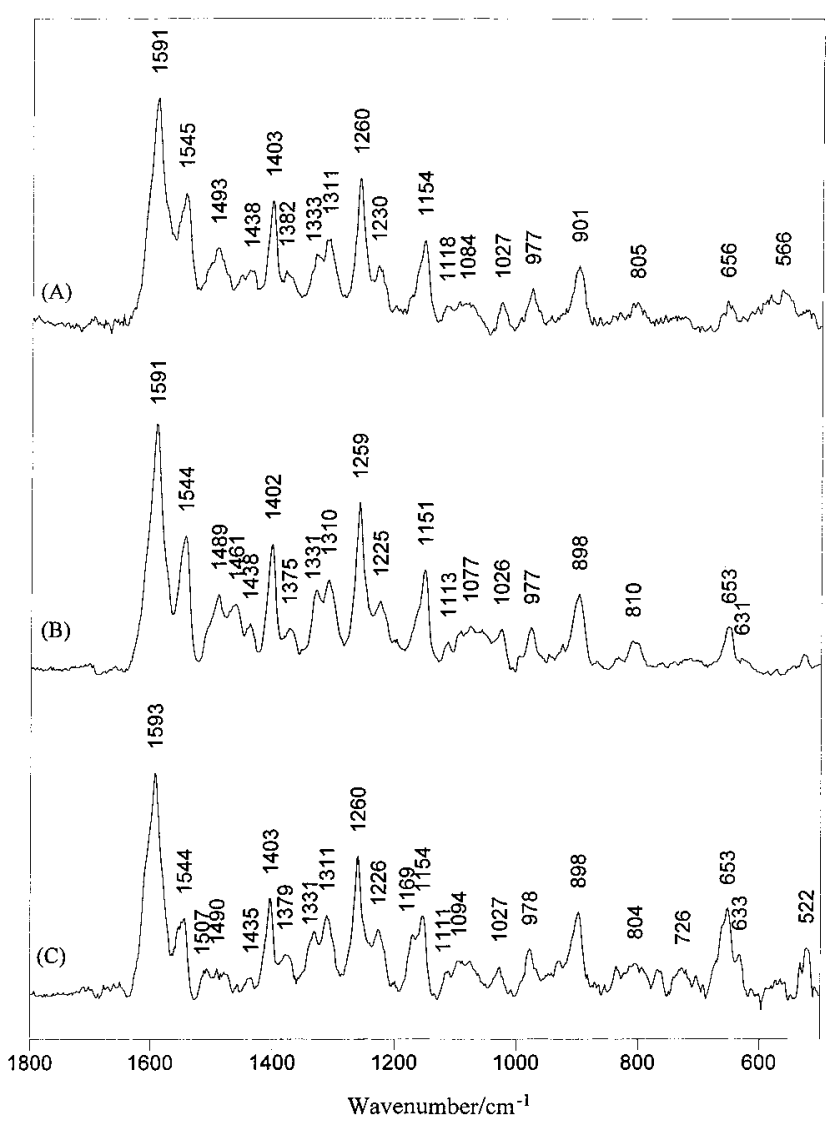

FIGURE 6: Raman spectra of 4-HBA-CoA bound to D145A mutant at various pHs: (A) 8.5; (B) 7.5; and (C) 7.0. All of the spectra were taken in $50 \mathrm{mM}$ Tris- $\mathrm{HCl}$ buffer.

Scheme 3: Electron Redistribution of 4-HBA-CoA in the D145S Mutant

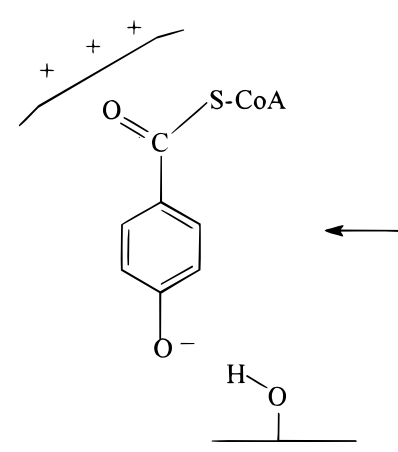

I phenolate form

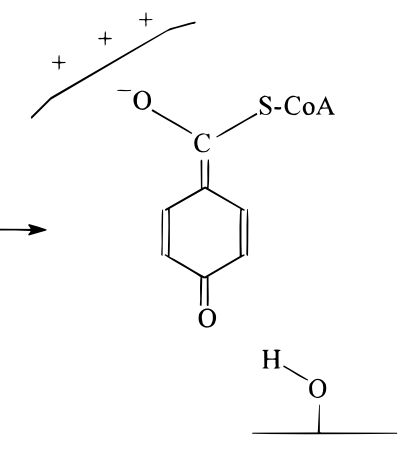

II quinone form
Raman difference data for the D145A complex at $\mathrm{pH}$ values of 8.5, 7.5, and 7.0 are presented in Figure 6. Attempts at recording spectra at lower $\mathrm{pH}$ were frustrated by the protein coming out of solution. At $\mathrm{pH} 8.5$ the spectrum is dominated by the same suite of bands as is found for the high pH D145S complex. These are at 1591, 1545, 1493, 1403,1260 , and $1153 \mathrm{~cm}^{-1}$. Interestingly, although the band positions are essentially identical for the D145A and D145S adducts, the relative intensities are different. Since peak positions are a property solely of the ground electronic state and intensities reflect both ground and excited state properties, the differences in relative intensities probably mean that there are differences in the electronic excited states associated with the benzoyl chromophore. One possible source of this difference may be that the proton on the serine side chain

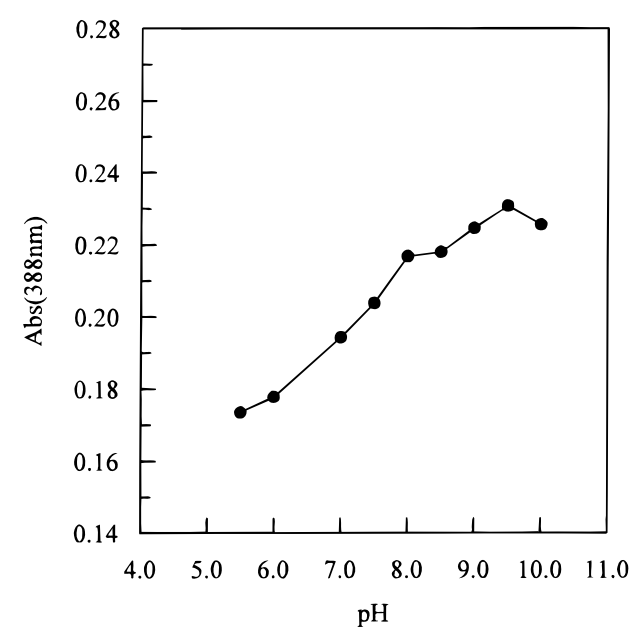

FIGURE 7: $\mathrm{pH}$ dependence of the absorbance of the $388 \mathrm{~nm}$ peak due to ionized 4-HBA-CoA on D145S dehalogenase.

can participate in the excited state in the D145S complex by migrating to the $4 \mathrm{O}^{-}$group, whereas no such possibility exists for the alanine mutant.

At pHs 7.5 and 8.5, there is no evidence for Raman bands due to the $4 \mathrm{OH}$ form in the D145A complex, but at $\mathrm{pH} 7.0$ this species is evidenced by a band at $1169 \mathrm{~cm}^{-1}$, increasing intensity near 1226, and the appearance of a shoulder near $1600 \mathrm{~cm}^{-1}$. Again this indicates that the protonationdeprotonation equilibrium exists in the active site. Both of the D145A and D145S complexes show the same characteristic change in the adenine triplet seen in the unbound ligand at 1378, 1341, and $1307 \mathrm{~cm}^{-1}$ in Figure 1.

Attempts at measuring an accurate $\mathrm{p} K_{\mathrm{a}}$ for the bound product in D145A and D145S were frustrated by the instability of dehalogenase solutions below $\mathrm{pH}$ 6.0-6.5. Moreover, using Raman data to derive the $\mathrm{p} K_{\mathrm{a}}$ is further complicated by the fact that the Raman scattering cross sections of the 4-OH and 4- $\mathrm{O}^{-}$forms are likely not the same; thus relative intensities from peaks from the two forms do not measure relative populations, directly. However, it was possible to arrive at a semiquantitative estimate of the $\mathrm{p} K_{\mathrm{a}}$. For D145S dehalogenase, inspection of Figure 5 shows evidence for an increasing population of the protonated form of the ligand going from $\mathrm{pH} 7.0$ to 6.5 , with little or no evidence for this form above $\mathrm{pH}$ 8.0. The absorption spectrum of the complex at $\mathrm{pH} 7.5$ shows a ratio of the band near $395 \mathrm{~nm}$, due to the ionized ligand, to the band at 260 $\mathrm{nm}$, due to the adenine ring and which is invariant to $\mathrm{pH}$ changes near neutral $\mathrm{pH}$, of approximately 2 (Figure 2 ). At $\mathrm{pH} 6.5$ this ratio drops to close to 1 . Figure 7 plots the intensity of the product absorbance at $388 \mathrm{~nm}$ versus $\mathrm{pH}$ for product bound to the D145S variant. The inability to obtain data below $\mathrm{pH} 6$ prevented the identification of a convincing low-pH plateau. However, the data in Figure 7 demonstrate that the $\mathrm{p} K_{\mathrm{a}}$ for ionization of the $4 \mathrm{OH}$ group is at 7.2 or lower. These factors taken together suggest the $\mathrm{p} K_{\mathrm{a}} \mathrm{s}$ for 4-HBA-CoA binding to D145S or D145A are in the region of $6.5-7.0$. This is approximately $1.5-2$ units below that found for free 4-HBA-CoA $(6,26)$ and demonstrates how the active site can manipulate electron density at the 4-position of the bound ligand, which is likely a prerequisite for the aromatic substitution reaction occurring at that point. 


\section{ACKNOWLEDGMENT}

The authors are grateful to the Ohio Supercomputer Center for computational facilities (project PDS 183).

\section{SUPPORTING INFORMATION AVAILABLE}

Density functional theory predicted normal modes for $S$-ethyl 4-oxybenzoate thioester anion (Table 2), and density functional theory predicted normal modes for $S$-ethyl 4-(dimethylamino)benzoate (Table 3 ). This material is available free of charge via the Internet at http://pubs.acs.org.

\section{REFERENCES}

1. Dunaway-Mariano, D., and Babbitt, P. C. (1994) Biodegradation 5, 259 .

2. Liu, R., Liang, P. H., Scholten, J., and Dunaway-Mariano, D. (1995) J. Am. Chem. Soc. 117, 5003.

3. Yang, G., Liang, P. H., and Dunaway-Mariano, D. (1994) Biochemistry 33, 8527.

4. Miller, J. (1968) in Reaction Mechanisms in Organic Chemistry (Eaborn, C., and Chapman, N. B., Eds.) Monograph 8, Chapman, Elsevier, New York.

5. Terrier, F. (1982) Chem. Rev. 82, 78.

6. Taylor, K. L., Liu, R., Liang, P. H., Price, J., DunawayMariano, D., Tonge, P. J., Clarkson, J., and Carey, P. R. (1995) Biochemistry 34, 13881.

7. Benning, M. M., Taylor, K. L., Liu, R., Yang, G., Xiang, H., Wesenberg, G., Dunaway-Mariano, D., and Holden, H. M. (1996) Biochemistry 35, 8103.

8. Taylor, K. L., Xiang, H., Liu, R.-Q., Yang, G., and DunawayMariano, D. (1997) Biochemistry 36, 1349.

9. Clarkson, J., Tonge, P. J., Taylor, K. L., Dunaway-Mariano, D., and Carey, P. R. (1997) Biochemistry 36, 10192.

10. Doran, J. D., and Carey, P. R. (1996) Biochemistry 35, 12495.

11. Xiang, H., Dong, J., Carey, P. R., and Dunaway-Mariano, D. (1999) Biochemistry 38, 4207-4213.

12. Kim, M., Owen, H., and Carey, P. R. (1993) Appl. Spectrosc. 47,1780 .
13. Dong, J., Dinakarpandian, D., and Carey, P. R. (1998) Appl. Spectrosc. 52, 1117.

14. Chang, K. H., Liang, P. H., Beck, W., Scholten, J. D., and Dunaway-Mariano, D. (1992) Biochemistry 31, 5605.

15. Liang, P. H., Yang, G., and Dunaway-Mariano, D. (1993) Biochemistry 32, 12245.

16. Xiang, H. (1998) Ph.D. Thesis, University of Maryland, College Park, MD.

17. Mieyel, J. J., Webster, L. T., and Siddiqui, U. A. (1974) J. Biol. Chem. 249, 2633.

18. Frisch, M. J., Trucks, G. W., Schlegel, H. B., Gill, P. M. W., Johnson, B. G., Robb, M. A., Cheeseman, J. R., Keith, T., Petersson, G. A., Montgomery, J. A., Raghavacharari, K., AlLaham, M. A., Zakrzewski, V. G., Ortiz, J. V., Foresman, J. B., Cioslowski, J., Stefanov, B. B., Nanayakkara, A., Challacombe, M., Peng, C. Y., Ayala, P. Y., Chen, W., Wong, M. W., Andres, J. L., Replogle, E. S., Gomperts, R., Martin, R. L., Fox, D. J., Binkley, J. S., Defrees, D. J., Baker, J., Stewart, J. P., Head-Gordon, M., Gonzalez, C., and Pople, J. A. (1995) Gaussian 94, Revision E.2., Gaussian, Inc., Pittsburgh, PA.

19. Scott, A. P., and Radom, L. (1996) J. Phys. Chem. 100, 16502.

20. Wilson, E. B. (1934) Phys. Rev. 45, 706.

21. Colthup, N. B., Daly, L. H., and Wiberly, S. E. (1975) Introduction to Infrared and Raman Spectroscopy, 2nd ed., Academic Press, New York.

22. Dollish, F. R., Fateley, W. G., and Bentley, F. F. (1973) Characteristic Raman Frequencies of Organic Compounds, John Wiley \& Sons, New York.

23. Varsanyi, G. (1969) Vibrational Spectra of Benzene Derivatives, Academic Press, New York.

24. Toyama, A., Takeuchi, H., and Harada, I. (1991) J. Mol. Struct. $242,2633$.

25. Nowak, M. J., Lapinski, L., Kwiatkowski, J. S., and Leszczynski, J. (1996) J. Phys. Chem. 100, 3527.

26. Webster, L. T., Mieyel, J. J., and Siddiqui, U. A. (1974) J. Biol. Chem. 249, 2641.

BI982668K 